\title{
Performance comparison of LFXLMS, MOVFXLMS and THF-NLFXLMS algorithms for Hammerstein NANC
}

\begin{abstract}
Filtered-X least mean square (FXLMS) algorithm is widely used in active noise control (ANC) systems when the secondary path is linear. However, the performance of FXLMS reduces when nonlinearity is present. Leaky FXLMS (LFXLMS) and minimum output variance FXLMS (MOVFXLMS) algorithms are effective in compensating the nonlinearity effects in nonlinear ANC (NANC). When using optimum leakage factors, these algorithms show close performance with benchmark nonlinear FXLMS (NLFXLMS) algorithm. In all three algorithms, the degree of nonlinearity must be known in advance and are usually assumed. In previous works, Tangential Hyperbolic Function NLFXLMS (THF-NLFXLMS) algorithm has been developed whereby the degree of nonlinearity is estimated using tangential hyperbolic function (THF). In this work, the performance of LFXLMS and MOVFXLMS based on optimum leakage factors calculated using the estimated degree of nonlinearity is compared with THF-NLFXLMS for Hammerstein structure. The results show that optimum MOVFXLMS performs similarly to optimum LFXLMS and THF-NLFXLMS.
\end{abstract}

Keyword: FXLMS; Hammerstein; LFXLMS; MOVFXLMS; NANC; Optimum leakage factor; THF-NLFXLMS 